\title{
The Rate at Which the Energy of Solutions for a Class of $p$-Laplacian Wave Equation Decays
}

\author{
Soufiane Mokeddem ${ }^{1}$ and Khaled Ben Walid Mansour ${ }^{2}$ \\ ${ }^{1}$ Laboratory of Biomathematics, Djillali Liabes University, PB 89, 22000 Sidi Bel Abbes, Algeria \\ ${ }^{2}$ Department of Mathematics, Djillali Liabes University, 22000 Sidi Bel Abbes, Algeria
}

Correspondence should be addressed to Soufiane Mokeddem; s_mokeddem@yahoo.com

Received 11 July 2015; Accepted 6 August 2015

Academic Editor: Ramón Quintanilla

Copyright (C) 2015 S. Mokeddem and K. B. W. Mansour. This is an open access article distributed under the Creative Commons Attribution License, which permits unrestricted use, distribution, and reproduction in any medium, provided the original work is properly cited.

We will investigate the decay estimate of the energy of the global solutions to the $p$-Laplacian wave equation with dissipation of the form $u_{t t}-\operatorname{div}\left(\left|\nabla_{x} u\right|^{p-2} \nabla_{x} u\right)+\sigma(t)\left(u_{t}-\operatorname{div}\left(\left|\nabla_{x} u_{t}\right|^{m-2} \nabla_{x} u_{t}\right)\right)=0$ under suitable assumptions on the positive function $\sigma$. For this end we use the multiplier method combined with nonlinear integral inequalities given by Martinez; the proof is based on the construction of a special weight function that depends on the behavior of $\sigma$.

\section{Introduction}

In this paper we are concerned with the energy decay rate of the $p$-Laplacian type wave equation of the form

$$
\begin{aligned}
& u_{t t}-\operatorname{div}\left(\left|\nabla_{x} u\right|^{p-2} \nabla_{x} u\right) \\
& +\sigma(t)\left(u_{t}-\operatorname{div}\left(\left|\nabla_{x} u_{t}\right|^{m-2} \nabla_{x} u_{t}\right)\right)=0 \\
& \text { in } \Omega \times[0,+\infty[\text {, } \\
& u=0 \quad \text { on } \Gamma \times[0,+\infty[\text {, } \\
& u(x, 0)=u_{0}(x), \\
& u_{t}(x, 0)=u_{1}(x)
\end{aligned}
$$

on $\Omega$,

where $\Omega$ is a bounded domain in $\mathbb{R}^{n}$ with smooth boundary $\Gamma=\partial \Omega, p, m \geq 2$ are real numbers, and $\sigma$ is a positive function satisfying some conditions to be specified later.

Problem $(P)$ can be considered as a system describing the longitudinal motion of a viscoelastic configuration obeying a nonlinear Voigt model. The bibliography of works in this direction is so long. We mention, for instance, the works of
Andrews [1], Andrews and Ball [2], Ang and Dinh [3], and Kawashima and Shibata [4].

Existence of global solution and the decay property of the energy for the wave equation related to the problem $(P)$ have been investigated by many authors through various approaches (see [5-8]).

In [5], Benaissa and Messaoudi considered the following problem:

$$
\begin{aligned}
u_{t t}-\Delta u+a\left(1+\left|u_{t}\right|^{m-2}\right) u_{t} & =-b u|u|^{p-2} \\
\quad \text { in } \Omega \times[0,+\infty[, & \quad \text { on } \Gamma \times[0,+\infty[, \\
u & =0 \\
u(x, 0) & =u_{0}(x), \\
u_{t}(x, 0) & =u_{1}(x)
\end{aligned}
$$

on $\Omega$,

where $a, b>0$. They showed that, for suitably chosen initial data, the problem has a global weak solution, which decays exponentially even if $m>2$. Further they proved the global existence by using the potential well theory introduced by Sattinger [9]. 
Similar results have been established by Ye $[7,10]$. In these works the author used the Faedo-Galerkin approximation together with compactness criteria and difference inequality introduced by Nakao [11].

More related studies of the nonlinear $p$-Laplacian wave equation type with damping term can be found in the papers $[6,8,12,13]$.

In [14], with considering $-\operatorname{div}\left(\left|\nabla_{x} u_{t}\right|^{m-2} \nabla_{x} u_{t}\right)$ instead of the damping term $\sigma(t)\left(u_{t}-\operatorname{div}\left(\left|\nabla_{x} u_{t}\right|^{m-2} \nabla_{x} u_{t}\right)\right)$, we have obtained global existence result by using the argument in [15] combined with the concepts of so-called stable sets due to Sattinger [9]. We have also shown the asymptotic behavior of global solutions through the use of the integral inequality given by Komornik [16].

The purpose of this paper is to give an energy decay estimate of the solution of problem $(P)$. Our proof is based on the multiplier method combined with nonlinear integral inequalities given by Martinez [17].

The plan of the paper is as follows. In the next section we present some assumptions, technical lemmas, and main result. Then in Section 3 we are devoted to the proof of decay estimate.

For simplicity of notation, we denote by $\|\cdot\|_{p}$ the Lebesgue space $L^{p}(\Omega)$ norm. In particular $\|\cdot\|_{2}$ denotes $L^{2}(\Omega)$ and $(\cdot, \cdot)$ the inner product of $L^{2}(\Omega)$. We also write equivalent norm $\|\nabla .\|_{p}$ instead of $W_{0}^{1, p}(\Omega)$ norm $\|\cdot\|_{W_{0}^{1, p}(\Omega)}$. As usual, we write, respectively, $u(t)$ and $u_{t}(t)$ instead of $u(x, t)$ and $u_{t}(x, t)$. Furthermore, throughout this paper the functions considered are all real valued.

\section{Preliminaries and Main Result}

First assume that $\sigma: \mathbb{R}_{+} \rightarrow \mathbb{R}_{+}$is a nonincreasing positive function of class $C^{1}$ on $\mathbb{R}_{+}$, satisfying

$$
\int_{0}^{+\infty} \sigma(\tau) d \tau=+\infty
$$

Let us define the energy equality associated with the solution of the problem $(P)$ by the following formula:

$$
E(t)=\frac{1}{2}\left\|u_{t}\right\|_{2}^{2}+\frac{1}{p}\|\nabla u\|_{p}^{p},
$$

for $u \in W_{0}^{1, p}(\Omega)$ and $t \geq 0$.

We state, without proof, a global existence result for the problem $(P)$. For more details we refer the reader to [6].

Theorem 1. Let $2 \leq m \leq p$ and assume that $\left(u_{0}, u_{1}\right) \in$ $W_{0}^{1, p}(\Omega) \times L^{2}(\Omega)$. Then, for any $T>0$, the problem $(P)$ has a unique strong solution $u(t)$ on $\Omega \times[0, T]$ in the class

$$
\begin{gathered}
u(t) \in L^{\infty}\left([0, T], W_{0}^{1, p}(\Omega)\right) \cap W^{1, \infty}\left([0, T], L^{2}(\Omega)\right) \\
\cap W^{1, m}\left([0, T], W_{0}^{1, m}(\Omega)\right) .
\end{gathered}
$$

We now present the following well-known lemmas which will be needed later.
Lemma 2 (energy identity). Let $u(x, t)$ be a global solution to the problem $(P)$ on $[0, \infty)$. Then one has

$$
\begin{aligned}
& E(t)+\int_{\Omega} \int_{0}^{t} \sigma(s) u_{t}\left(u_{t}-\operatorname{div}\left(\left|\nabla_{x} u_{t}\right|^{m-2} \nabla_{x} u_{t}\right)\right) d s d x \\
& =E(0)
\end{aligned}
$$

for all $t \in[0, \infty)$.

Lemma 3 (Sobolev-Poincaré inequality). Let $p \in \mathbb{N}^{*}$ and $r \in$ $\mathbb{R}$ with $2 \leq r<+\infty(n=1,2, \ldots, p)$ or $2 \leq r \leq n p /(n-$ p) $(n \geq p+1)$. Then there is a constant $c_{*}=c_{*}(\Omega, p, r)$ such that

$$
\|u\|_{r} \leq c_{*}\|\nabla u\|_{p} \quad \text { for } u \in W_{0}^{1, p}(\Omega) .
$$

The case $p=r=2$ gives the known Poincarés inequality.

Before stating our main result, we introduce the following lemma which plays an important part in studying the decay estimate of energy associated with the solution of the problem $(P)$.

Lemma 4 (see [17]). Let $E: \mathbb{R}_{+} \rightarrow \mathbb{R}_{+}$be a nonincreasing function and $\phi: \mathbb{R}_{+} \rightarrow \mathbb{R}_{+}$an increasing $C^{2}$ function such that

$$
\begin{aligned}
& \phi(0)=0, \\
& \phi(t) \longrightarrow+\infty \text { as } t \longrightarrow+\infty .
\end{aligned}
$$

Assume that there exist $q \geq 0$ and $\gamma>0$ such that

$$
\int_{S}^{+\infty} E(t)^{q+1} \phi^{\prime}(t) d t \leq \gamma^{-1} E(0)^{q} E(S)
$$

$$
0 \leq S<+\infty \text {. }
$$

Then one has

$$
\begin{aligned}
& E(t) \leq E(0)\left(\frac{1+q}{1+q \gamma \phi(t)}\right)^{1 / q} \quad \forall t \geq 0 \text { if } q>0, \\
& E(t) \leq E(0) \exp (1-\gamma \phi(t)) \quad \forall t \geq 0 \text { if } q=0 .
\end{aligned}
$$

Now we are in position to state and prove our main result.

Theorem 5. Let $\left(u_{0}, u_{1}\right) \in W_{0}^{1, p}(\Omega) \times L^{2}(\Omega)$ and $p \geq m \geq 2$. Suppose that (2) holds. Then the solution $u(x, t)$ of the problem $(P)$ satisfies the following energy decay estimates.

(1) If $p=m$, then there exists a positive constant $\omega$ such that

$E(t) \leq c(E(0)) \exp \left(1-\omega \int_{0}^{t} \sigma(\tau) d \tau\right) \quad \forall t>0$.

(2) If $p>m$, then there exists a positive constant $c(E(0))$ depending continuously on E(0) such that

$$
E(t) \leq\left(\frac{c(E(0))}{\int_{0}^{t} \sigma(\tau) d \tau}\right)^{p(m-1) /(p-m)} \quad \forall t>0 .
$$




\section{Proof of Main Result}

From now on, we denote by $c$ various positive constants depending on the known constants and they may be different at each appearance.

Multiplying by $E^{q} \phi^{\prime}(t) u$ on both sides of the first equation of $(P)$ and integrating over $\Omega \times[T, S]$, where $\phi$ is a function satisfying all the hypotheses of Lemma 4 and $0 \leq$ $S \leq T \leq+\infty$, we obtain that

$$
\begin{aligned}
0= & \int_{S}^{T} E^{q} \phi^{\prime} \int_{\Omega} u\left[u_{t t}-\operatorname{div}\left(\left|\nabla_{x} u\right|^{p-2} \nabla_{x} u\right)\right. \\
& \left.+\sigma(t)\left(u_{t}-\operatorname{div}\left(\left|\nabla_{x} u_{t}\right|^{m-2} \nabla_{x} u_{t}\right)\right)\right] d x d t .
\end{aligned}
$$

By an integration by parts we see that

$$
\begin{array}{r}
0=\left[E^{q} \phi^{\prime} \int_{\Omega} u u_{t}\right]_{S}^{T}-\int_{S}^{T}\left(q E^{\prime} E^{q-1} \phi^{\prime}+E^{q} \phi^{\prime \prime}\right) \\
\quad \cdot \int_{\Omega} u u_{t} d x d t-\int_{S}^{T} E^{q} \phi^{\prime} \int_{\Omega}\left|u_{t}\right|^{2} d x d t \\
+\int_{S}^{T} E^{q} \phi^{\prime} \int_{\Omega}|\nabla u|^{p} d x d t+\int_{S}^{T} E^{q} \phi^{\prime} \sigma(t) \\
+\int_{\Omega} u\left(u_{t}-\operatorname{div}\left(\left|\nabla_{x} u_{t}\right|^{m-2} \nabla_{x} u_{t}\right)\right) d x d t .
\end{array}
$$

Hence from the definition of energy and a simple argument we can obtain

$$
\begin{aligned}
& p \int_{S}^{T} E^{q+1} \phi^{\prime} d t=-\left[E^{q} \phi^{\prime} \int_{\Omega} u u_{t}\right]_{S}^{T} \\
& \quad+\int_{S}^{T}\left(q E^{\prime} E^{q-1} \phi^{\prime}+E^{q} \phi^{\prime \prime}\right) \int_{\Omega} u u_{t} d x d t+\left(\frac{p}{2}+1\right) \\
& \quad \cdot \int_{S}^{T} E^{q} \phi^{\prime} \int_{\Omega}\left|u_{t}\right|^{2} d x d t-\int_{S}^{T} E^{q} \phi^{\prime} \sigma \\
& \quad \cdot \int_{\Omega} u\left(u_{t}-\operatorname{div}\left(\left|\nabla_{x} u_{t}\right|^{m-2} \nabla_{x} u_{t}\right)\right) d x d t .
\end{aligned}
$$

Now we must estimate both sides of (14) to arrive at a similar inequality as (8).

Define

$$
\phi(t)=\int_{0}^{t} \sigma(\tau) d \tau
$$

It is clear that $\phi$ is a nondecreasing function of class $C^{2}$ on $\mathbb{R}_{+}$ and hypothesis (2) ensures that

$$
\phi(t) \longrightarrow+\infty \quad \text { as } t \longrightarrow+\infty \text {. }
$$

Since $E$ is nonincreasing, $\phi^{\prime}$ is a bounded nonnegative function on $\mathbb{R}_{+}$(we denote by $\mu$ its maximum) and, using the definition of energy, Cauchy-Schwartz inequality, and Sobolev-Poincaré inequality, we have

$$
-\left|\left[E^{q} \phi^{\prime} \int_{\Omega} u u_{t} d x\right]_{S}^{T}\right| \leq c \mu E(S)^{q+1 / 2+1 / p}
$$

where the above estimate follows from the fact that

$$
\begin{aligned}
\int_{\Omega} u u_{t} d x & \leq\|u\|_{2}\left\|u_{t}\right\|_{2} \leq c\|\nabla u\|_{p}\left\|u_{t}\right\|_{2} \\
& \leq c E(t)^{1 / p} E(t)^{1 / 2} .
\end{aligned}
$$

Again, exploiting Cauchy-Schwartz inequality, SobolevPoincaré inequality, the definition of energy, and (18), we obtain

$$
\begin{aligned}
& \left|\int_{S}^{T}\left(q E^{\prime} E^{q-1} \phi^{\prime}+E^{q} \phi^{\prime \prime}\right) \int_{\Omega} u u_{t} d x d t\right| \\
& \leq c \mu \int_{S}^{T}-E^{\prime} E^{q-1 / 2+1 / p} d t \\
& \quad+\int_{S}^{T} c E^{q+1 / 2+1 / p}\left(-\phi^{\prime \prime}\right) d t \leq c \mu E(S)^{q+1 / 2+1 / p}
\end{aligned}
$$

where the fact that $E(t)$ is nonincreasing is used.

Furthermore, by using Lemma 2, we have

$$
\begin{aligned}
(1 & \left.+\frac{p}{2}\right) \int_{S}^{T} E^{q} \phi^{\prime} \int_{\Omega}\left|u_{t}\right|^{2} d x d t \\
& \leq\left(1+\frac{p}{2}\right) \int_{S}^{T} E^{q} \phi^{\prime} \int_{\Omega}\left(\left|u_{t}\right|^{2}+\left|\nabla u_{t}\right|^{m}\right) d x d t \\
& \leq\left(1+\frac{p}{2}\right) \int_{S}^{T} E^{q} \phi^{\prime}\left(-\frac{E^{\prime}(t)}{\sigma(t)}\right) d t \leq c E^{q+1}(S) .
\end{aligned}
$$

We then estimate the last term in (14) as follows:

$$
\begin{aligned}
& \left|\int_{S}^{T} E^{q} \phi^{\prime} \sigma(t) \int_{\Omega} u\left(u_{t}-\operatorname{div}\left(\left|\nabla_{x} u_{t}\right|^{m-2} \nabla_{x} u_{t}\right)\right) d x d t\right| \\
& \leq\left|\int_{S}^{T} E^{q} \phi^{\prime} \sigma(t) \int_{\Omega} u u_{t} d x d t\right| \\
& \quad+\left|\int_{S}^{T} E^{q} \phi^{\prime} \sigma(t) \int_{\Omega} u \operatorname{div}\left(\left|\nabla_{x} u_{t}\right|^{m-2} \nabla_{x} u_{t}\right) d x d t\right|,
\end{aligned}
$$

which implies that

$$
\begin{gathered}
\left|\int_{S}^{T} E^{q} \phi^{\prime} \sigma(t) \int_{\Omega} u \operatorname{div}\left(\left|\nabla_{x} u_{t}\right|^{m-2} \nabla_{x} u_{t}\right) d x d t\right| \\
\quad \leq \int_{S}^{T} E^{q} \phi^{\prime} \sigma(t) \int_{\Omega}|\nabla u|\left|\nabla u_{t}\right|^{m-1} d x d t .
\end{gathered}
$$

Using Hölder's and Sobolev-Poincaré's inequalities, we see that

$$
\begin{gathered}
\left|\int_{S}^{T} E^{q} \phi^{\prime}(t) \sigma(t) \int_{\Omega} u \operatorname{div}\left(\left|\nabla_{x} u_{t}\right|^{m-2} \nabla_{x} u_{t}\right) d x d t\right| \\
\quad \leq \int_{S}^{T} E^{q} \phi^{\prime} \sigma(t)\|\nabla u\|_{p}\left\|\nabla u_{t}\right\|_{p(m-1) /(p-1)}^{m-1} d t .
\end{gathered}
$$

We also have

$$
\begin{gathered}
\left\|\nabla u_{t}\right\|_{p(m-1) /(p-1)}^{m-1} \leq c|\Omega|^{(p-m) / p m}\left\|\nabla u_{t}\right\|_{m}^{m-1}, \\
\left\|\nabla u_{t}\right\|_{m}^{m-1} \leq\left(\frac{-E^{\prime}(t)}{\sigma(t)}\right)^{(m-1) / m} .
\end{gathered}
$$


This gives

$$
\begin{aligned}
& \left|\int_{S}^{T} E^{q} \phi^{\prime}(t) \sigma(t) \int_{\Omega} u \operatorname{div}\left(\left|\nabla_{x} u_{t}\right|^{m-2} \nabla_{x} u_{t}\right)\right| \\
& \quad \leq c \int_{S}^{T} E^{q+1 / p} \phi^{\prime} \sigma(t)\left(\frac{-E^{\prime}(t)}{\sigma(t)}\right)^{(m-1) / m} d t \\
& \quad=c \int_{S}^{T} E^{q+1 / p} \phi^{\prime} \sigma(t)^{1 / m}\left(-E^{\prime}(t)\right)^{(m-1) / m} d t .
\end{aligned}
$$

Further, by Young inequality, we have for $\varepsilon>0$

$$
\begin{gathered}
\left|\int_{S}^{T} E^{q} \phi^{\prime}(t) \sigma(t) \int_{\Omega} u \operatorname{div}\left(\left|\nabla_{x} u_{t}\right|^{m-2} \nabla_{x} u_{t}\right)\right| \\
\leq \frac{1}{m} \varepsilon^{m} \int_{S}^{T} E^{(q+1 / p) m}\left(\phi^{\prime}(t)\right)^{m} \sigma(t) d t \\
\quad+\frac{m-1}{m} \frac{1}{\varepsilon(m /(m-1))} E(S) \\
=\frac{1}{m} \varepsilon^{m} \int_{S}^{T} E^{(q+1 / p) m} \phi^{\prime}(t)(\sigma(t))^{m} d t \\
+\frac{m-1}{m} \frac{1}{\varepsilon(m /(m-1))} E(S) .
\end{gathered}
$$

We choose $q$ such that

$$
m\left(q+\frac{1}{p}\right)=q+1 .
$$

Thus $q=(p-m) / p(m-1)$.

Combining estimates (17)-(26), (14) becomes

$$
\begin{aligned}
& \int_{S}^{T} E^{q+1} \phi^{\prime}(t) d t \\
& \quad \leq c E(S)^{q+1 / 2+1 / p}+c^{\prime} E(S)^{q+1}+c^{\prime \prime} E(S) \\
& \quad \leq\left(\frac{c E(0)^{q-1 / 2+1 / p}+c^{\prime} E(0)^{q}+c^{\prime \prime}}{E(0)^{q}}\right) E(0)^{q} E(S),
\end{aligned}
$$

where $c, c^{\prime}$, and $c^{\prime \prime}$ are different positive constants independent of $E(0)$.

Letting $T \rightarrow+\infty$, this yields the following estimate:

$$
\begin{aligned}
& \int_{S}^{+\infty} E^{q+1} \phi^{\prime}(t) d t \\
& \quad \leq\left(\frac{c E(0)^{q-1 / 2+1 / p}+c^{\prime} E(0)^{q}+c^{\prime \prime}}{E(0)^{q}}\right) E(0)^{q} E(S),
\end{aligned}
$$

$\forall S \geq 0$,

and we conclude from Lemma 4 that

$$
\begin{aligned}
E(t) \leq & \left(c E(0)^{q-1 / 2+1 / p}+c^{\prime} E(0)^{q}+c^{\prime \prime}\right)^{1 / q} \\
& \cdot\left(\frac{1+q}{q}\right)^{1 / q} \int_{0}^{t} \sigma(t) d t .
\end{aligned}
$$

It is clear that, for $p=m$, we have $q=0$ and the energy $E(t)$ associated with the solution of the problem $(P)$ satisfies the decay property in (10).

\section{Conflict of Interests}

The authors declare that there is no conflict of interests regarding the publication of this paper.

\section{References}

[1] G. Andrews, "On the existence of solutions to the equation $u_{t t}=$ $u_{x x t}+\sigma\left(u_{x}\right)_{x}$," Journal of Differential Equations, vol. 35, no. 2, pp. 200-231, 1980.

[2] G. Andrews and J. M. Ball, "Asymptotic behaviour and changes of phase in one-dimensional nonlinear viscoelasticity," Journal of Differential Equations, vol. 44, no. 2, pp. 306-341, 1982.

[3] D. D. Ang and P. N. Dinh, "Strong solutions of a quasilinear wave equation with nonlinear damping," SIAM Journal on Mathematical Analysis, vol. 19, no. 2, pp. 337-347, 1988.

[4] S. Kawashima and Y. Shibata, "Global existence and exponential stability of small solutions to nonlinear viscoelasticity," Communications in Mathematical Physics, vol. 148, no. 1, pp. 189-208, 1992.

[5] A. Benaissa and S. A. Messaoudi, "Exponential decay of solutions of a nonlinearly damped wave equation," Nonlinear Differential Equations and Applications, vol. 12, no. 4, pp. 391399, 2005.

[6] Y. Zhijian, "Existence and asymptotic behaviour of solutions for a class of quasi-linear evolution equations with non-linear damping and source terms," Mathematical Methods in the Applied Sciences, vol. 25, no. 10, pp. 795-814, 2002.

[7] Y.-J. Ye, "On the decay of solutions for some nonlinear dissipative hyperbolic equations," Acta Mathematicae Applicatae Sinica, vol. 20, no. 1, pp. 93-100, 2004.

[8] Y. Ye, "Exponential decay of energy for some nonlinear hyperbolic equations with strong dissipation," Advances in Difference Equations, vol. 2010, Article ID 357404, 12 pages, 2010.

[9] D. H. Sattinger, "On global solution of nonlinear hyperbolic equations," Archive for Rational Mechanics and Analysis, vol. 30, no. 2, pp. 148-172, 1968.

[10] Y. Ye, "Existence of global solutions for some nonlinear hyperbolic equation with a nonlinear dissipative term," Journal of Zhengzhou University, vol. 29, no. 3, pp. 18-23, 1997.

[11] M. Nakao, "A difference inequality and its application to nonlinear evolution equations," Journal of the Mathematical Society of Japan, vol. 30, no. 4, pp. 747-762, 1978.

[12] N.-E. Amroun and S. Mimouni, "Asymptotic behaviour of solutions for some weakly dissipative wave equations of $\mathrm{p}$ Laplacian type," Applied Mathematics E-Notes, vol. 11, pp. 175183, 2011.

[13] A. Benaissa and S. Mokeddem, "Decay estimates for the wave equation of $\mathrm{p}$-Laplacian type with dissipation of m-Laplacian type," Mathematical Methods in the Applied Sciences, vol. 30, no. 2, pp. 237-247, 2007.

[14] S. Mokeddem and K. B. W. Mansour, "Asymptotic behaviour of solutions for $p$-Laplacian wave equation with $m$-Laplacian dissipation," Zeitschrift für Analysis und ihre Anwendungen, vol. 33, no. 3, pp. 259-269, 2014. 
[15] Y. Ye, "Global existence and asymptotic behavior of solutions for some nonlinear hyperbolic equation," Journal of Inequalities and Applications, vol. 2010, Article ID 895121, 10 pages, 2010.

[16] V. Komornik, Exact Controllability and Stabilization. The Multiplier Method, Research in Applied Mathematics, John Wiley, Chichester, UK, 1994.

[17] P. Martinez, "A new method to decay rate estimates for dissipative systems," ESAIM: Control, Optimisation and Calculus of Variations, vol. 4, pp. 419-444, 1999. 


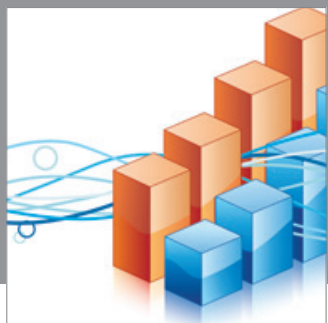

Advances in

Operations Research

mansans

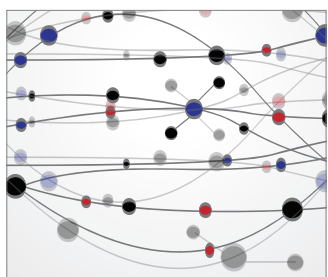

The Scientific World Journal
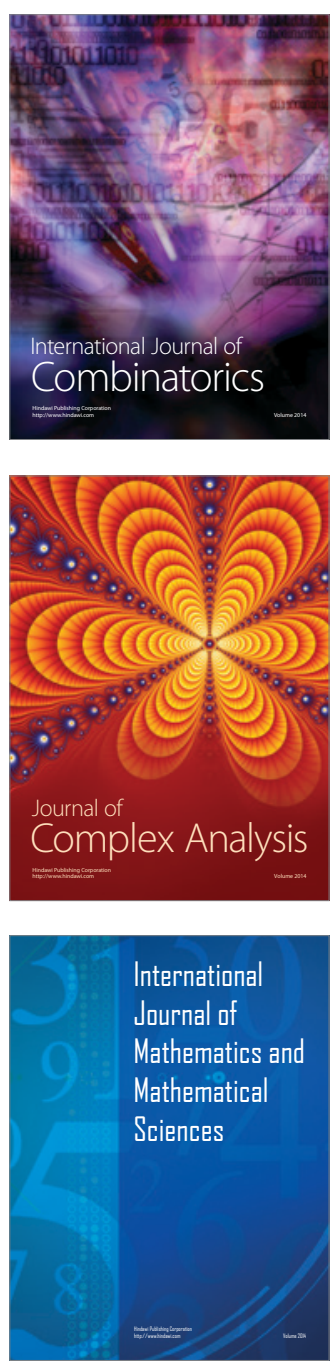
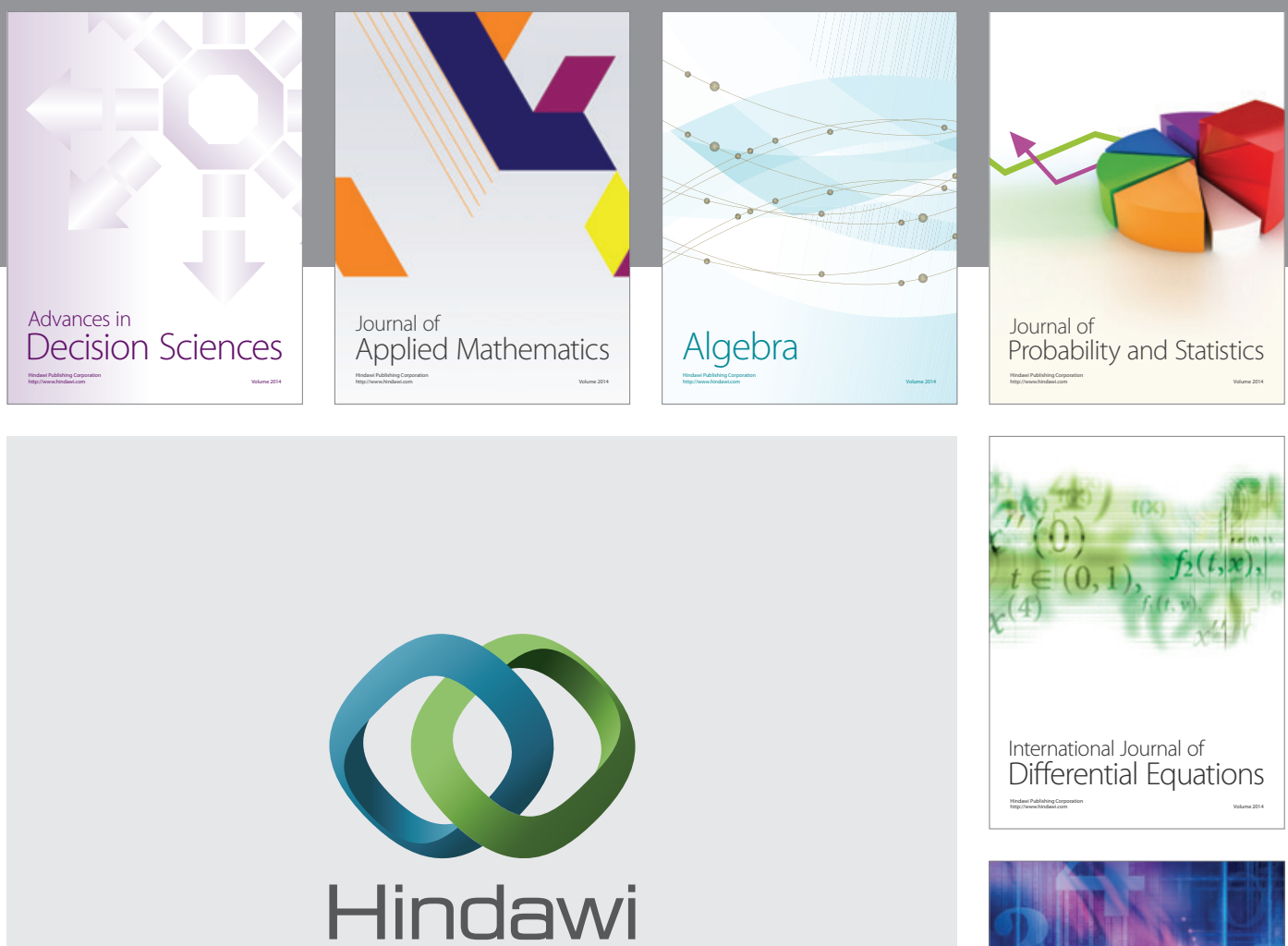

Submit your manuscripts at http://www.hindawi.com
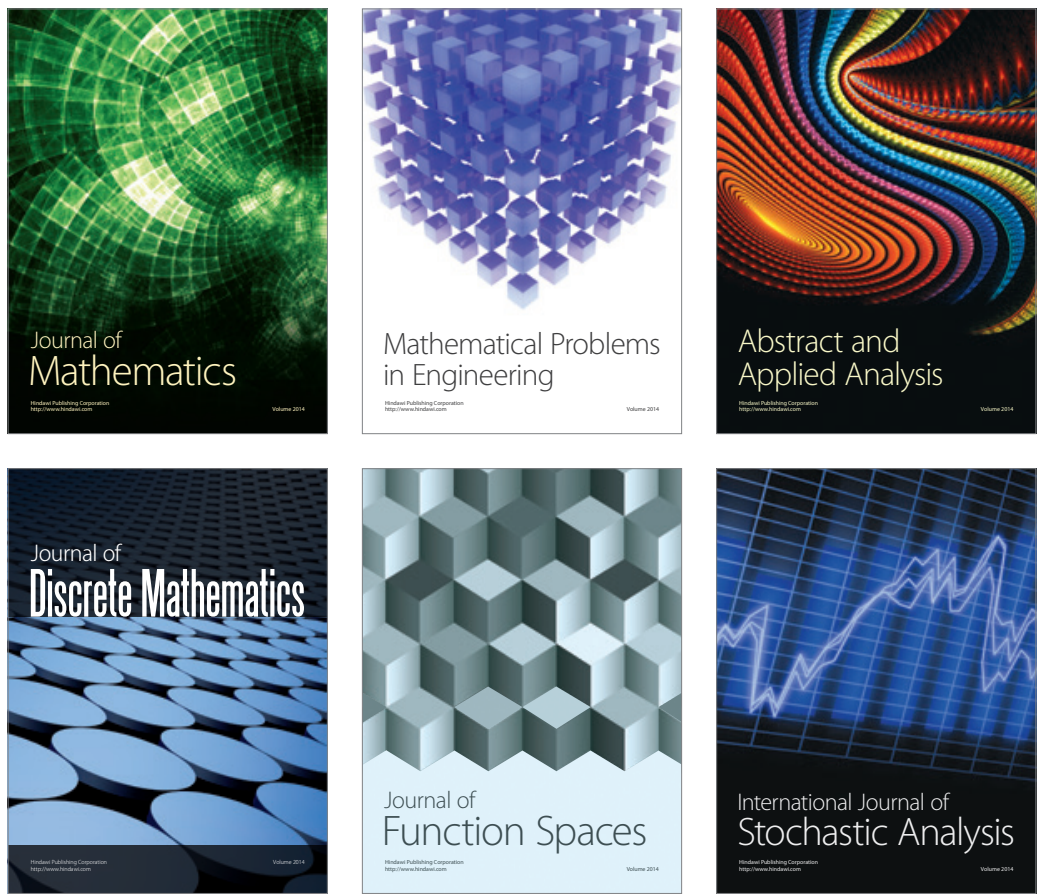

Journal of

Function Spaces

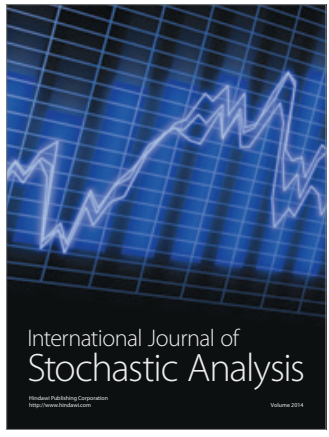

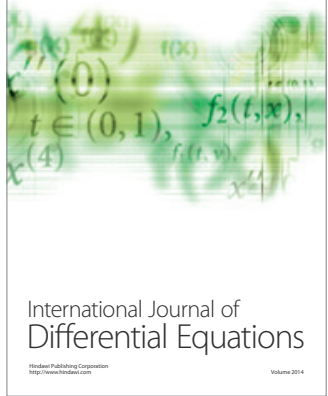
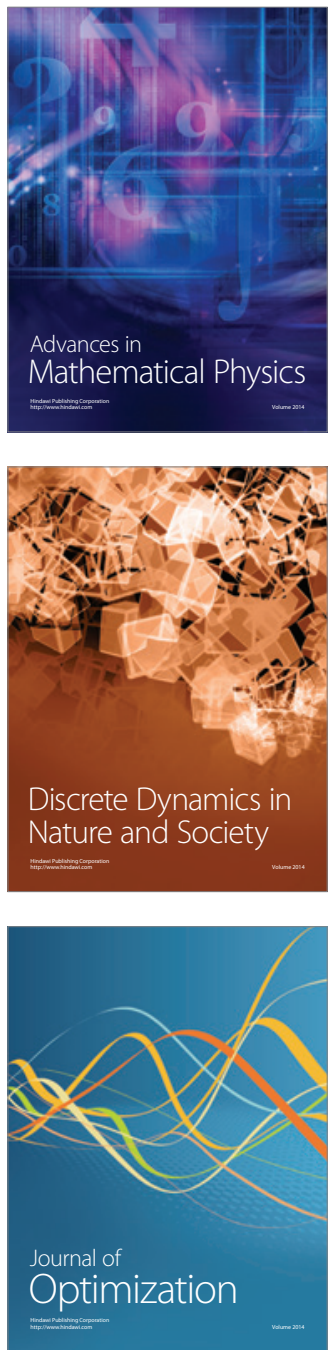